\title{
Testing Lorentz symmetry violation with an invariant minimum speed
}

\author{
Cláudio Nassif*, A. C. Amaro de Faria, Jr. and Rodrigo Francisco dos Santos \\ CPFT - Centro de Pesquisas em Física Teórica, Rua Rio de Janeiro 1186/s.1304, Lourdes, \\ CEP:30.160-041, Belo Horizonte-MG, Brazil \\ Instituto de Estudos Avançados, Rodovia dos Tamoios Km 099, \\ São José dos Campos-SP, CEP:12.220-000, Brazil \\ Universidade Federal Fluminense, Av. Litoranea s/n, Gragoatá, \\ CEP:24210-340, Niterói-RJ, Brazil \\ *claudionassif@yahoo.com.br
}

Received 13 June 2018

Accepted 4 July 2018

Published 17 July 2018

This work presents an experimental test of Lorentz invariance violation in the infrared (IR) regime by means of an invariant minimum speed in spacetime and its effects on the time when an atomic clock given by a certain radioactive single-atom (e.g. isotope $\mathrm{Na}^{25}$ ) is a thermometer for an ultracold gas like the dipolar gas $\mathrm{Na}^{23} \mathrm{~K}^{40}$. So, according to a Deformed Special Relativity (DSR) so-called Symmetrical Special Relativity (SSR), where there emerges an invariant minimum speed $V$ in the subatomic world, one expects that the proper time of such a clock moving close to $V$ in thermal equilibrium with the ultracold gas is dilated with respect to the improper time given in lab, i.e. the proper time at ultracold systems elapses faster than the improper one for an observer in the lab, thus leading to the so-called proper time dilation so that the atomic decay rate of an ultracold radioactive sample (e.g. $\mathrm{Na}^{25}$ ) becomes larger than the decay rate of the same sample at room temperature. This means a suppression of the half-life time of a radioactive sample thermalized with an ultracold cloud of dipolar gas to be investigated by NASA in the Cold Atom Lab (CAL).

Keywords: Lorentz violation; minimum speed; proper time dilation; Planck mass.

PACS No.: 11.30.Qc

\section{Introduction}

In the last 20 years, the scientific community has shown an intense interest in the theories that contained and investigated the breakdown of Lorentz symmetry in

* Corresponding author

This is an Open Access article published by World Scientific Publishing Company. It is distributed under the terms of the Creative Commons Attribution 4.0 (CC-BY) License. Further distribution of this work is permitted, provided the original work is properly cited. 
many scenarios ${ }^{1-12}$ and also the so-called Deformed Special Relativities (DSR), ${ }^{13,14}$ although no relevant experimental fact has demonstrated Lorentz symmetry breaking until the present time. However, there could be the evidence that the breakdown of Lorentz symmetry may exist in a lower energy regime. In this paper, we propose an experimental investigation of this fact in view of a possible existence of a non-null minimum speed $V$ that has been postulated in a previous work ${ }^{15}$ and incorporated into the so-called Symmetrical Special Relativity (SSR) theory, where there should be two invariant speeds, namely the speed of light $c$ (the maximum speed) for higher energies, being related to a maximum temperature (Planck temperature $T_{\mathrm{P}} \sim 10^{32} \mathrm{~K}$ ) within the cosmological scenario (early universe), and the minimum speed $V\left(\sim 10^{-14} \mathrm{~m} / \mathrm{s}\right)^{15}$ for lower energies, being related to a minimum temperature. However, due to the unattainable minimum speed $V$ for representing the zero energy of a particle, the new dynamics in SSR subtly change the conception about what should mean a minimum temperature in the cosmological scenario, which will be denominated as the Planck scale of temperature in SSR, i.e. $T_{\min }<T<T_{\mathrm{P}}$ as it will be investigated later.

The theory of SSR is one of the most recent DSRs and it has many far-reaching consequences. For instance, the Heisenberg uncertainty principle that acquires an origin from the spacetime of $\mathrm{SSR}^{17}$ and the tiny positive value of the cosmological constant connected to the low vacuum energy density were elucidated by SSR. ${ }^{15-17}$ Also, an intriguing consequence of this minimum speed is the symmetry between the proper and improper time, where both can either suffer contraction or dilation. This issue is investigated in this paper with the purpose of establishing an experimental route of detecting a new time effect when the speed $v$ is close to the minimum speed $V$ connected to a minimum temperature, ${ }^{18}$ by taking into account an ultracold gas in thermal equilibrium with a sample of radioactive atoms.

In fact, the striking implication concerning the existence of the minimum speed $V$ is the breaking of Lorentz invariance, since $V$ establishes the existence of a preferential reference frame for a background field by representing the vacuum energy related to the cosmological constant within a de Sitter scenario. ${ }^{19}$

Due to the wide implications of a background field connected to $V$, ranging from quantum mechanics ${ }^{17}$ to cosmological models ${ }^{15}$ with a potential of application in quantum gravity, important experiments attempting to either confirm or rule out this hypothesis would be very acknowledged. Then, here one proposes an accessible experiment in view of the current improvement of the technology of ultracold systems that has the goal of reaching temperatures in the order of $10^{-10} \mathrm{~K}$ in CAL.

\section{A Brief Review of Symmetrical Special Relativity}

Here, some basic results of the Symmetrical Special Relativity (SSR) ${ }^{15}$ are presented. According to SSR, a new status of referential is defined, namely the so-called ultra-referential $S_{V}$, i.e. the background reference frame. In SSR, the idea of ultrareferential is connected to the invariant minimum speed $V$, thus being unattainable 


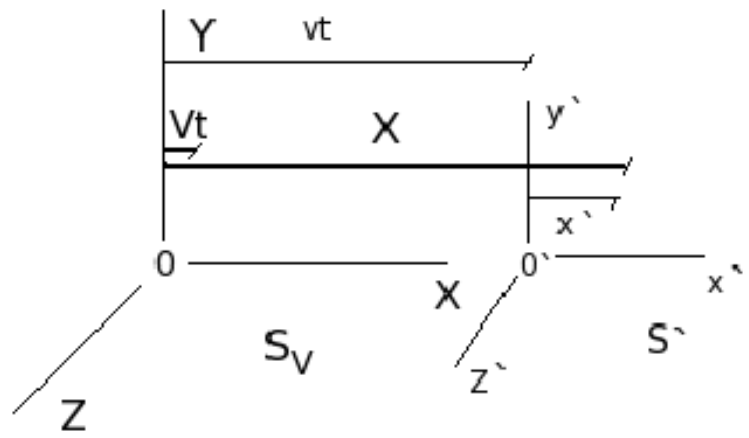

Fig. 1. In this special case $(1+1) D$, the referential $S^{\prime}$ moves in $x$-direction with a speed $v(>V)$ with respect to the background field connected to the ultra-referential $S_{V}$. If $V \rightarrow 0, S_{V}$ is eliminated (empty space), and thus the galilean frame $S$ takes place, recovering the Lorentz transformations.

by any particle with speed $v>V$. Thus, SSR provides the so-called non-Galilean reference frames, since such a given frame can be thought of as being a set of all the particles having a given speed $v$ with respect to the own ultra-referential $S_{V}$ of the background field (Fig. 1).

The three postulates of SSR are as follows.

(1) Non-equivalence (asymmetry) of the non-Galilean reference frames due to the presence of the background frame $S_{V}$ that breaks down the Lorentz symmetry, i.e. we cannot change $v$ for $-v$ by means of inverse transformations in spacetime as we can never achieve the rest state for a certain non-Galilean reference frame in order to reverse the direction of its velocity just for one spatial dimension.

(2) The invariance of the speed of light $(c)$.

(3) The covariance of the ultra-referential $S_{V}$ (the background framework) connected to an invariant minimum limit of speed $(V)$.

The $(1+1) \mathrm{D}$-transformations in $\mathrm{SSR}^{15-18}$ with $\mathbf{v}=v_{x}=v$ (Fig. 1) are

$$
x^{\prime}=\Psi(X-v t+V t)=\theta \gamma(X-v t+V t)
$$

and

$$
t^{\prime}=\Psi\left(t-\frac{v X}{c^{2}}+\frac{V X}{c^{2}}\right)=\theta \gamma\left(t-\frac{v X}{c^{2}}+\frac{V X}{c^{2}}\right),
$$

where the factor $\theta=\sqrt{1-V^{2} / v^{2}}$ and $\Psi=\theta \gamma=\sqrt{1-V^{2} / v^{2}} / \sqrt{1-v^{2} / c^{2}}$.

The $(3+1) D$-transformations in SSR were shown in a previous paper, $\frac{15}{15}$ namely:

$$
\mathbf{r}^{\prime}=\theta\left[\mathbf{r}+(\gamma-1) \frac{(\mathbf{r} \cdot \mathbf{v})}{v^{2}} \mathbf{v}-\gamma \mathbf{v}(1-\alpha) t\right],
$$


where $\alpha=V / v$, and

$$
t^{\prime}=\theta \gamma\left[t-\frac{\mathbf{r} \cdot \mathbf{v}}{c^{2}}(1-\alpha)\right]
$$

From Eqs. (3) and (4), we can verify that, if we consider $\mathbf{v}$ to be in the same direction of $\mathbf{r}$, with $r=X$, we recover the special case of $(1+1)$ D-transformations given by Eqs. (1) and (2).

The breaking of Lorentz symmetry group in SSR destroys the properties of the transformations of Special Relativity (SR) and so generates an intriguing deformed kinematics and dynamics for speeds very close to the minimum speed $V$, i.e. for $v \rightarrow V$. Thus, close to $V$, the relativistic effects to the reverse are found such as the contraction of the improper time and the dilation of space. ${ }^{15}$ In this new scenario, proper time also suffers relativistic effects such as its own dilation with respect to improper time when $v \approx V$, thus leading to $\Delta \tau>\Delta t$. So it was obtained ${ }^{15,16}$

$$
\Delta \tau \sqrt{1-\frac{V^{2}}{v^{2}}}=\Delta t \sqrt{1-\frac{v^{2}}{c^{2}}}
$$

from where it will be made an experimental prospect for detecting the new relativistic effect of improper time contraction (or proper time dilation) close to the minimum speed $V\left(=\sqrt{G m_{e} m_{p}} e / \hbar \cong 4.58 \times 10^{-14} \mathrm{~m} / \mathrm{s}\right)^{15}$ that breaks Lorentz symmetry at very low energies. We have $e=q_{e} / \sqrt{4 \pi \epsilon_{0}}$, where $q_{e}$ is the electron charge. $m_{e}$ is the electron mass and $m_{p}$ is the proton mass.

We should stress that the minimum speed $V$ was obtained in a previous paper ${ }^{15}$ by taking into account the well-known Dirac's large number hypothesis (LNH), where we have the ratio $F_{e} / F_{g}=q_{e}^{2} / 4 \pi \epsilon_{0} G m_{e} m_{p} \sim 10^{40}$.

The structure of spacetime in SSR generates a new effect on mass (energy), being symmetrical to what happens close to the speed of light $c$, i.e. it was shown that $E=m_{0} c^{2} \Psi(v)=m_{0} c^{2} \sqrt{1-V^{2} / v^{2}} / \sqrt{1-v^{2} / c^{2}}$, so that $E \rightarrow 0$ when $v \rightarrow V$. It is noticed that $E=E_{0}=m_{0} c^{2}$ for $v=v_{0}=\sqrt{c V}$. The momentum is $p=$ $m_{0} v \Psi(v)=m_{0} v \sqrt{1-V^{2} / v^{2}} / \sqrt{1-v^{2} / c^{2}}$ and the deformed dispersion relation is $E^{2}=c^{2} p^{2}+m_{0}^{2} c^{4}\left(1-V^{2} / v^{2}\right) \cdot 15,16$

The spacetime metric of SSR is a deformed Minkowski metric with the scale factor $\Theta^{15}$ working like a conformal metric, ${ }^{19}$ as follows:

$$
d s^{2}=\Theta \eta_{\mu \nu} d x^{\mu} d x^{\nu}
$$

where $\eta_{\mu \nu}$ is the Minkowski metric and $\Theta$ is a conformal factor.

In a recent paper,$\frac{19}{19}$ it was shown that SSR-metric in Eq. (6) is equivalent to a conformal metric given in spherical coordinates with a set of negative curvatures represented by a set of positive cosmological constants $\Lambda$ working like a cosmological scalar field within a de Sitter (dS) scenario, namely:

$$
d s^{2}=\frac{c^{2} d t^{2}}{\left(1-\frac{\Lambda r^{2}}{6 c^{2}}\right)^{2}}-\frac{d r^{2}}{\left(1-\frac{\Lambda r^{2}}{6 c^{2}}\right)^{2}}-\frac{r^{2} d \Omega}{\left(1-\frac{\Lambda r^{2}}{6 c^{2}}\right)^{2}},
$$


where $d \Omega=(d \theta)^{2}+\sin ^{2} \theta(d \Phi)^{2}$ is the solid angle and the conformal factor is $\Theta=\theta^{-2}=1 /\left(1-V^{2} / v^{2}\right) \equiv 1 /\left(1-\Lambda r^{2} / 6 c^{2}\right)^{2} \underline{19}$ with coordinate dependence.

\subsection{Energy barrier of the minimum speed connected to the vacuum energy: The idea of dressed mass}

Let us consider a force applied to a particle in the same direction of its motion. More general cases where the force is not necessarily parallel to velocity will be treated elsewhere. In our specific case $(\mathbf{F} \| \mathbf{v})$, the relativistic power $P_{\text {ow }}(=v d p / d t)$ of SSR is given as follows:

$$
P_{\mathrm{ow}}=v \frac{d}{d t}\left[m_{0} v\left(1-\frac{V^{2}}{v^{2}}\right)^{\frac{1}{2}}\left(1-\frac{v^{2}}{c^{2}}\right)^{-\frac{1}{2}}\right],
$$

where we have used the momentum in SSR, i.e. $p=m_{0} v \Psi(v)$.

After performing the calculations in Eq. (8), we find

$$
P_{\mathrm{ow}}=\left[\frac{\left(1-\frac{V^{2}}{v^{2}}\right)^{\frac{1}{2}}}{\left(1-\frac{v^{2}}{c^{2}}\right)^{\frac{3}{2}}}+\frac{V^{2}}{v^{2}\left(1-\frac{v^{2}}{c^{2}}\right)^{\frac{1}{2}}\left(1-\frac{V^{2}}{v^{2}}\right)^{\frac{1}{2}}}\right] \frac{d E_{k}}{d t},
$$

where $E_{k}=\frac{1}{2} m_{0} v^{2}$.

If we make $V \rightarrow 0$ and $c \rightarrow \infty$ in Eq. (9), we simply recover the power obtained in Newtonian mechanics, namely $P_{\text {ow }}=d E_{k} / d t$. Now, if we just consider $V \rightarrow 0$ in Eq. (9), we recover the well-known relativistic power of SR, namely $P_{\mathrm{ow}}=$ $\left(1-v^{2} / c^{2}\right)^{-3 / 2} d E_{k} / d t$. We notice that such a relativistic power tends to infinite $\left(P_{\text {ow }} \rightarrow \infty\right)$ in the limit $v \rightarrow c$. We explain this result as an effect of the drastic increase of an effective inertial mass close to $c$, namely $m_{\mathrm{eff}}=m_{0}\left(1-v^{2} / c^{2}\right)^{k^{\prime \prime}}$, where $k^{\prime \prime}=-3 / 2$. We must stress that such an effective inertial mass is the response to an applied force parallel to the motion according to Newton's second law, and it increases faster than the relativistic mass $m=m_{r}=m_{0}\left(1-v^{2} / c^{2}\right)^{-1 / 2}$.

The effective inertial mass $m_{\text {eff }}$ that we have obtained is a longitudinal mass $m_{L}$, i.e. it is a response to the force applied in the direction of motion. In SR, for the case where the force is perpendicular to velocity, we can show that the transversal mass increases like the relativistic mass, i.e. $m=m_{T}=m_{0}\left(1-v^{2} / c^{2}\right)^{-1 / 2}$, which differs from the longitudinal mass $m_{L}=m_{0}\left(1-v^{2} / c^{2}\right)^{-3 / 2}$. So, in this sense, there is anisotropy of the effective inertial mass to be also investigated in more details by SSR in a further work.

The mysterious discrepancy between the relativistic mass $m\left(m_{r}\right)$ and the longitudinal inertial mass $m_{L}$ from Newton's second law (Eq. (9)) is a controversial issue. ${ }^{20-25}$ Actually the Newtonian notion about inertia as the resistance to an acceleration $\left(m_{L}\right)$ is not compatible with the relativistic dynamics $\left(m_{r}\right)$ in the sense that we generally cannot consider $\mathbf{F}=m_{r} \mathbf{a}$. The dynamics of SSR aims to give us a new interpretation for the inertia of the Newtonian point of view in order to make 
it compatible with the relativistic mass. This compatibility is possible only due to the influence of the background field that couples to the particle and "dresses" its relativistic mass in order to generate an effective (dressed) mass in accordance with the Newtonian notion about inertia from Eqs. (8) and (9). This issue will be clarified in this section.

From Eq. (9), it is important to observe that, when we are closer to $V$, there emerges a completely new result (correction) for power, as

$$
P_{\mathrm{ow}} \approx\left(1-\frac{V^{2}}{v^{2}}\right)^{-\frac{1}{2}} \frac{d}{d t}\left(\frac{1}{2} m_{0} v^{2}\right),
$$

given in the approximation $v \approx V$. So, we notice that $P_{\text {ow }} \rightarrow \infty$ when $v \approx V$. We can also make the limit $v \rightarrow V$ for the general case (Eq. (9)) and so we obtain an infinite power $\left(P_{\mathrm{ow}} \rightarrow \infty\right)$. Such a new relativistic effect deserves the following very important comment: Although we are in the limit of very low energies close to $V$, where the energy of the particle $\left(m c^{2}\right)$ tends to zero according to the approximation $E=m c^{2} \approx m_{0} c^{2}\left(1-V^{2} / v^{2}\right)^{k}$ with $k=1 / 2$, on the other hand, the power given in Eq. (10) shows us that there is an effective inertial mass that increases to infinite in the limit $v \rightarrow V$, that is to say, from Eq. (10) we get the effective mass $m_{\mathrm{eff}} \approx$ $m_{0}\left(1-V^{2} / v^{2}\right)^{k^{\prime}}$, where $k^{\prime}=-1 / 2$. Therefore, from a dynamical point of view, the negative exponent $k^{\prime}(=-1 / 2)$ for the power at very low speeds (Eq. (10)) is responsible for the inferior barrier of the minimum speed $V$, as well as the exponent $k^{\prime \prime}=-3 / 2$ of the well-known relativistic power is responsible for the top barrier of the speed of light $c$ according to Newton's second law (Fig. 2). Actually, in view of the drastic increase of $m_{\text {eff }}$ of a particle moving closer to $S_{V}$, leading to its strong coupling to the vacuum field in the background frame $S_{V}$, the dynamics of SSR

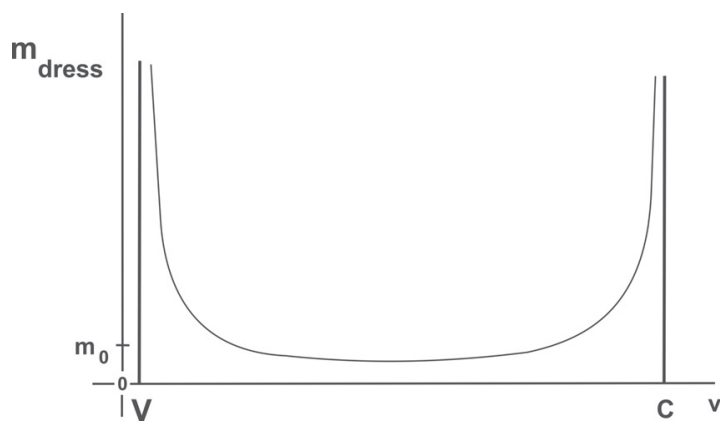

Fig. 2. The graph shows us two infinite barriers at $V$ and $c$, providing an aspect of symmetry of SSR. The first barrier $(V)$ is exclusively due to the vacuum- $S_{V}$, being interpreted as a barrier of pure vacuum energy. In this regime, we have the following approximations: $m_{\mathrm{eff}}=m_{\mathrm{dressed}} \approx$ $\Delta m_{i} \approx m_{0}\left(1-V^{2} / v^{2}\right)^{-1 / 2}$ and $m_{r} \approx m_{0}\left(1-V^{2} / v^{2}\right)^{1 / 2}$, so that $m_{\text {dressed }} \rightarrow \infty$ and $m=m_{r}=$ $m_{\text {bare }} \rightarrow 0$ when $v \rightarrow V$. The second barrier $(c)$ is a sum (mixture) of two contributions, namely the own bare (relativistic) mass $m$ that increases with the factor $\gamma=\left(1-v^{2} / c^{2}\right)^{-1 / 2}$ plus the interactive increment $\Delta m_{i}$ due to the vacuum energy- $S_{V}$, so that $m_{\text {dressed }}=m_{L}=m+\Delta m_{i} \approx$ $m_{0}\left(1-v^{2} / c^{2}\right)^{-3 / 2}$. This is a longitudinal effect. For the transversal effect, $\Delta m_{i}=0$ since we get $m_{T}=m$. This result will be shown elsewhere. 
states that it is impossible to decelerate a subatomic particle until reaching the rest. This is the reason why there is an unattainable minimum speed $V$.

In order to see clearly both exponents $k^{\prime}=-1 / 2$ (inferior inertial barrier $V$ ) and $k^{\prime \prime}=-3 / 2$ (top inertial barrier $c$ ), let us write the general formula of power (Eq. (9)) in the following alternative way after some algebraic manipulations on it, as

$$
P_{\mathrm{ow}}=\left(1-\frac{V^{2}}{v^{2}}\right)^{k^{\prime}}\left(1-\frac{v^{2}}{c^{2}}\right)^{k^{\prime \prime}}\left(1-\frac{V^{2}}{c^{2}}\right) \frac{d E_{k}}{d t},
$$

where $k^{\prime}=-1 / 2$ and $k^{\prime \prime}=-3 / 2$. Now it is easy to see that, if $v \approx V$ or even $v \ll c$, Eq. (11) recovers the approximation in Eq. (10). As $V \ll c$, the ratio $V^{2} / c^{2}(\ll 1)$ in Eq. (11) is a very small dimensionless constant $\xi^{2}=V^{2} / c^{2} \sim 10^{-44}$. $\frac{15}{\text {. So }} \xi^{2}$ can be neglected in Eq. (11).

So, from Eq. (11) we get the effective inertial mass $m_{\text {eff }}$ of SSR, as

$$
m_{\mathrm{eff}}=m_{0}\left(1-\frac{V^{2}}{v^{2}}\right)^{-\frac{1}{2}}\left(1-\frac{v^{2}}{c^{2}}\right)^{-\frac{3}{2}} .
$$

We must stress that $m_{\text {eff }}$ in Eq. (12) is a longitudinal mass $m_{L}$. The problem of mass anisotropy will be treated elsewhere. But here we intend to show that, just for the approximation $v \approx V$, the effective inertial mass becomes practically isotropic, that is to say $m_{L} \approx m_{T} \approx m_{0}\left(1-\frac{V^{2}}{v^{2}}\right)^{-1 / 2}$. This important result will show us the isotropic aspect of the vacuum- $S_{V}$, so that the inferior barrier $V$ has the same behavior of response $\left(k^{\prime}=-1 / 2\right)$ of a force applied at any direction in space, namely for any angle between the applied force and the velocity of the particle.

We must point out the fact that $m_{\text {eff }}$ has nothing to do with the "relativistic mass" (relativistic energy $E=m_{0} c^{2} \Psi(v)$ ) in the sense that $m_{\text {eff }}$ is dynamically responsible for both barriers $V$ and $c$. The discrepancy between the "relativistic mass" (energy $m c^{2}$ of the particle) and such an effective inertial mass $\left(m_{\text {eff }}\right)$ can be interpreted under SSR theory, as follows: $m_{\text {eff }}$ is a dressed inertial mass given in response to the presence of the vacuum- $S_{V}$ that works like a kind of "fluid" in which the particle $m_{0}$ is immersed, while the "relativistic mass" in SSR $\left(m=m_{\text {rel. }}=m_{0} \Psi(v)\right)$ works like a bare inertial mass in the sense that it is not considered to be under the dynamical influence of the "fluid" connected to the vacuum- $S_{V}$. That is the reason why the exponent $k=1 / 2$ cannot be used to explain the existence of an infinite barrier at $V$, such as the vacuum- $S_{V}$ barrier which is governed by the exponent $k^{\prime}=-1 / 2$ as shown in Eqs. (10)-(12), and which prevents $v_{*}(=v-V) \leq 0$.

The difference between the dressed (effective) mass and the relativistic (bare) mass, i.e. $m_{\mathrm{eff}}-m$ represents an interactive increment of mass $\Delta m_{i}$, that has purely origin from the vacuum energy of $S_{V}$, as:

$$
\Delta m_{i}=m_{0}\left[\frac{\left(1-\frac{V^{2}}{c^{2}}\right)}{\left(1-\frac{V^{2}}{v^{2}}\right)^{\frac{1}{2}}\left(1-\frac{v^{2}}{c^{2}}\right)^{\frac{3}{2}}}-\frac{\left(1-\frac{V^{2}}{v^{2}}\right)^{\frac{1}{2}}}{\left(1-\frac{v^{2}}{c^{2}}\right)^{\frac{1}{2}}}\right] .
$$


We have $\Delta m_{i}=m_{\mathrm{eff}}-m$, where $m_{\mathrm{eff}}=m_{\mathrm{dressed}}$ is given in Eq. (12) and $m\left(m_{r}=m_{0} \Psi\right)$ is the relativistic mass in SSR.

From Eq. (13), we consider the following special cases.

(a) For $v \approx c$ we have

$$
\Delta m_{i} \approx m_{0}\left[\left(1-\frac{v^{2}}{c^{2}}\right)^{-\frac{3}{2}}-\left(1-\frac{v^{2}}{c^{2}}\right)^{-\frac{1}{2}}\right] .
$$

As the effective inertial mass $m_{\text {eff }}\left(m_{L}\right)$ increases much faster than the bare (relativistic) mass $m\left(m_{r}\right)$ close to the speed $c$, there is an increment of inertial mass $\Delta m_{i}$ that dresses $m$ in direction of its motion and it tends to be infinite when $v \rightarrow c$, i.e. $\Delta m_{i} \rightarrow \infty$.

(b) For $V \ll v \ll c$ (Newtonian or intermediary regime) we find $\Delta m_{i} \approx 0$, where we simply have $m_{\text {eff }}\left(m_{\text {dressed }}\right) \approx m \approx m_{0}$. This is the classical approximation.

(c) For $v \approx V$ (close to the vacuum- $S_{V}$ regime), we have the following approximation:

$$
\Delta m_{i}=\left(m_{\mathrm{dressed}}-m\right) \approx m_{\mathrm{dressed}} \approx \frac{m_{0}}{\sqrt{1-\frac{V^{2}}{v^{2}}}},
$$

where $m=m_{0} \Psi \approx 0$ when $v \approx V$.

The approximation in Eq. (15) shows that the whole dressed mass has purely originated from the energy of vacuum- $S_{V}$, with $m_{\text {dressed }}$ being the pure increment $\Delta m_{i}$, since the bare (relativistic) mass $m$ of the own particle almost vanishes in such a regime $(v \approx V)$, and thus an inertial effect only due to the vacuum ("fluid")- $S_{V}$ remains. We see that $\Delta m_{i} \rightarrow \infty$ when $v \rightarrow V$. In other words, we can interpret this infinite barrier of vacuum- $S_{V}$ by considering the particle to be strongly coupled to the background field- $S_{V}$ in all directions of the space. The isotropy of $m_{\text {eff }}$ in this regime will be shown in detail elsewhere, being $m_{\text {eff }}=m_{L}=m_{T} \approx m_{0}\left(1-V^{2} / v^{2}\right)^{-1 / 2}$. In such a regime, the particle practically loses its locality ("identity") in the sense that it is spread out isotropically in the whole space and it becomes strongly coupled to the vacuum field- $S_{V}$, leading to an infinite value of $\Delta m_{i}$. Such a divergence of the dressed mass has origin from the dilation factor $\Theta_{v}(\rightarrow \infty)$ for this regime when $v \approx V$, so that we can rewrite Eq. (15) in the following way: $\Delta m_{i} \approx m_{\text {dressed }} \approx m_{0} \Theta(v)^{1 / 2}$. That is essentially the dynamical explanation why the particle cannot reach the rest in SSR theory so that the background frame of the vacuum- $S_{V}$ becomes unattainable for any particle. However, in the macroscopic (classical) level, the minimum speed $V$ as well as the Planck constant $\hbar$ are neglectable as a good approximation, such that the rest state is naturally recovered in spite of the fact that the subatomic particles that constitute a macroscopic object at rest are always moving, since its temperature can neither reach absolute zero, $\frac{18}{2}$ nor can their constituent subatomic particles ever reach $V$. 


\section{The Proper Time Dilation of an Atomic Clock in an Ultracold Gas}

Let us consider an ultracold gas so that the root-mean-square speed per atom (molecule) of the gas is close to the minimum speed $V$, i.e. in this special case, all the "atoms" ("molecules") are found in the same state with speed $v$ close to $V$, so that one can write $\sqrt{\left\langle v^{2}\right\rangle}=v \rightarrow V$. Here, it must be stressed that when one is too close to such a universal minimum speed, the idea of an individual atom (molecule) with mass $m_{0}$ in a gas does not make sense, as one is very close to the vacuum regime working like a kind of superfluid (condensate) represented by a "super-atom" that plays the role of a super-particle of vacuum with Planck mass $\left(M_{\mathrm{P}}\right)$, i.e. a kind of dark energy could be produced in such an ultra-special condition of too low temperatures as expected to occur in CAL. And SSR comes to predict a change in passing of the time for this regime of Lorentz violation, i.e. we find Eq. (5) given in the approximation $v \approx V\left(\Delta \tau \approx \Delta t \theta^{-1}=\Delta t\left(1-V^{2} / v^{2}\right)^{-1 / 2}\right)$.

In order to justify the $M_{\mathrm{P}}$ for representing the mass of the "super-atom" (superparticle) as being the set of all the strongly correlated particles given in a regime too close to the minimum speed $V$, let us think that the strong correlation between the particles so close to $V$ can be explained by the increasing of the dressed mass of any particle of the gas, so that we consider the existence of an upper cut-off for the dressed mass, being associated with $M_{\mathrm{P}} \sim 10^{-8} \mathrm{~kg}$, i.e. $M_{\mathrm{P}} \sim m_{\text {dressed }}=$ $m_{0} \theta^{-1}(v \approx V)$ (Eq. (15)), since we should have in mind that the Planck energy $E_{\mathrm{P}}=M_{\mathrm{P}} c^{2} \sim 10^{19} \mathrm{GeV}$ is the highest scale of energy within the cosmological scenario. So, we should note that $M_{\mathrm{P}}$ is the standard universal mass connected to vacuum.

The dilation of the proper time increases the persistence of correlation between two points inside the condensate and thus keeps the synchronization of two atomic clocks even out of the thermal equilibrium where a very rapid energy dissipation occurs between both points (atomic clocks) as shown recently by Pigneur et al. ${ }^{27}$ who claim that such a persistence of correlation out of the equilibrium is maintained by a mechanism still unknown and whose origin could be elucidated by means of the proper time dilation of both atomic clocks inside the condensate. Thus, the spacetime of SSR seems to be the foundation to explain the experimental evidence reported in the recent Ref. 27 , but this subject deserves a more careful investigation elsewhere.

Later it will be realized that there are two extreme energy regimes where matter is condensed into two types of vacuum, one of them with very high temperature (Planck energy $M_{\mathrm{P}} c^{2}$ for the minimum length of Planck $L_{\mathrm{P}}$ ) and the other one with a very low temperature to be investigated as consequence of SSR, where such a lowest speed $V$ would be related to a very low temperature close to the absolute zero temperature, which is considered to be in accordance with the classical kinematics like Newtonian or even relativistic mechanics, where there exist rest connected to the absolute zero temperature. Such inconsistency between classical 
motion (rest) and the absolute zero temperature, which is prevented by the third law of thermodynamics, can be solved by SSR, since the non-classical mechanics of SSR with an unattainable minimum speed $V$ could justify dynamically the impossibility of reaching the absolute zero temperature. $\frac{18}{}$ But, on the other hand, one should be pointed out the fact that the absolute zero temperature would mean a complete absence of motion as well as an infinite temperature would be related to an infinite speed, which must be replaced by the finite speed of light $c$. In this sense, the absolute zero temperature as the absence of motion does not seem to be compatible with the existence of a non-null minimum speed $V$ according to SSR, which is able to introduce a compatibility between the lowest speed $V$ and a lowest non-null temperature $\left(T_{\min }\right)$ by correcting by a lower cut-off the absolute zero as well as it is already known that the speed of light $c$ comes to replace an infinite speed, and an infinite temperature by an upper cut-off of maximum temperature so-called Planck temperature $T_{\mathrm{P}}\left(\sim 10^{32} \mathrm{~K}\right)$.

Therefore, SSR also introduces two cut-offs of temperature and energy in the cosmological scenario, namely one of them is well-known as the Planck energy in the early universe related to the Planck length $L_{\mathrm{P}}$, i.e. a UV cut-off $\left(E_{\mathrm{P}}=M_{\mathrm{P}} c^{2}\right)$, and the other one would be related to a minimum energy density of vacuum connected to a horizon (maximum) length $L_{\Lambda}$ for a given cosmological constant $(\Lambda)$, i.e. a IR cutoff given by $\operatorname{SSR}\left(E_{\min }=M_{\mathrm{P}} V^{2}\right)$, as it was initially investigated by Padmanabhan ${ }^{26}$ and recently advanced by SSR that has introduced an invariant minimum speed in order to provide a kinematics explanation for the cosmological constant ${ }^{15,16}$ and also for dS-horizon ${ }^{19} L_{\Lambda}$ connected to $E_{\text {min }}$ for representing the most cold cosmological vacuum. In order to perceive this as a classical picture, imagine a super-particle of vacuum with mass $M_{\mathrm{P}}$ having a minimum vibration $V$. On the other hand, $E_{\mathrm{P}}\left(\sim 10^{19} \mathrm{GeV}\right)$ represents the most hot cosmological vacuum (early universe) with the strongest repulsive field well-known as inflaton, but this issue should be deeply treated by using the formalism of energy-momentum tensor in SSR elsewhere.

The theory of SSR establishes a symmetry of both speeds and temperatures, so that we have $V<v<c$ for representing $T_{\min }<T<T_{\mathrm{P}}$, where such a minimum temperature $T_{\min }$ should be determined by SSR, since there should be a connection between a unattainable nonzero temperature $T_{\min }$ and the new universal constant of minimum speed $V$. Now it is easy to realize that such connection is obtained analogously to that already obtained for the maximum temperature $T_{\mathrm{P}}$ and $c$, where $T_{\mathrm{P}}=M_{\mathrm{P}} c^{2} / K_{\mathrm{B}}\left(\sim 10^{32} \mathrm{~K}\right)$, with $M_{\mathrm{P}}\left(=\sqrt{\hbar c / G} \cong 2.176 \times 10^{-8} \mathrm{~kg}\right)$ being the Planck mass and $K_{\mathrm{B}}\left(\cong 1.38 \times 10^{-23} \mathrm{~J} / \mathrm{K}\right)$ the Boltzmann constant. As it is known that $T_{\mathrm{P}} \propto c^{2}$, due to the symmetry of both limits of speed in SSR, it is expected that $T_{\min } \propto V^{2}$, so that it is found that $T_{\min }=M_{\mathrm{P}} V^{2} / K_{\mathrm{B}}\left(\cong 3.28 \times 10^{-12} \mathrm{~K}\right)$ and thus one obtains the ratio $T_{\min } / T_{\mathrm{P}}=V^{2} / c^{2} \sim 10^{-44}$. In view of this minimum ratio and by considering a gas with temperature $T$, two following fundamental equivalence relations are obtained: 


$$
\frac{V^{2}}{\left\langle v^{2}\right\rangle}=\frac{T_{\min }}{T}
$$

so that $T>T_{\min }$ for $\sqrt{\left\langle v^{2}\right\rangle}>V$.

Here, it must be stressed that $T_{\min }$ comes to replace the classical idea of an absolute zero by an absolute non-null vibration due to the invariant minimum speed $V$, so that now one cannot find $T \leq T_{\min }$ or even $v \leq V$ within the cosmological scenario with temperature. Thus SSR allows to define a cosmological (absolute) scale of temperature where the lowest temperature is of the order of $10^{-12} \mathrm{~K}$. Let us call it as the Planck scale of temperature in SSR.

On the other hand, it is easy to obtain

$$
\frac{\left\langle v^{2}\right\rangle}{c^{2}}=\frac{T}{T_{\mathrm{P}}}
$$

where we have $T<T_{\mathrm{P}}$ for $\sqrt{\left\langle v^{2}\right\rangle}<c$.

Here, it must be also stressed that one considers the cosmological scale of temperature according to the Standard Model (SM) since the maximum temperature is the Planck temperature $\left(T_{\mathrm{P}}\right)$ that unifies all the four forces of nature. On the other hand, the minimal temperature $\left(T_{\min } \propto V^{2}\right)$ that has origin in a quantum gravity at lower energies close to $V$ is beyond SM of cosmology due to its direct connection with the dark energy and also the inflationary field (inflaton), but this subject should be explored elsewhere.

As only the new effect of proper time dilation close to the minimum speed $V$ or its equivalent minimum temperature $T_{\min }\left(=M_{\mathrm{P}} V^{2} / K_{\mathrm{B}} \sim 10^{-12} \mathrm{~K}\right)$ could be tested in CAL (a Lorentz violation in the infrared regime), we make the following approximation of vacuum $\left(m_{\mathrm{dressed}} \approx M_{\mathrm{P}}\right)$ given for gases with much lower temperatures in Eq. (5), i.e. we just consider $T \approx T_{\min }\left(\sqrt{\left\langle v^{2}\right\rangle} \approx V\right)$, so that we just take into account Eq. (16) into Eq. (5) and thus we neglect Eq. (17) $\left(T / T_{\mathrm{P}} \approx 0\right)$, by simply writing Eq. (5) in the following form:

$$
\tau \approx \frac{t}{\sqrt{1-\frac{V^{2}}{\left\langle v^{2}\right\rangle}}}=\frac{t}{\sqrt{1-\frac{T_{\min }}{T}}},
$$

where $T_{\min }=M_{\mathrm{P}} V^{2} / K_{\mathrm{B}}$, with $\theta \equiv \theta(T)=\sqrt{1-\frac{T_{\min }}{T}}$.

We have $\tau$, which is the proper time of an atomic clock (atom of a radioactive sample) inside the ultracold gas (condensate), and $t$ is the improper time given in the lab at room temperature.

\subsection{Suppression of the half-life time}

By considering initially $N_{0}$ atoms of a radioactive sample that begins to decay, then, the sample will acquire a number $N$ of atoms after the time $t$ in lab, such a sample will acquire a number $N$ of atoms, so that

$$
N=N_{0} \exp (-\lambda t)
$$


where $\lambda$ is the so-called disintegration constant of a given radioactive sample. The time $t_{1 / 2}$ that leads to $N=N_{0} / 2$ is well-known as the half-life time and it is given by $t_{1 / 2}=\ln (2) / \lambda$. Normally, the half-life time depends only on the type of radioactive sample given by a certain disintegration constant $\lambda$, since one considers that the proper time $\tau$ of an atomic clock inside an ultracold sample is equal to the improper one $t$ in the lab. However, it will be shown that $t_{1 / 2}$ is suppressed by lower temperatures due to the dilation of the proper time $(\tau>t)$ according to Eq. (18) that reveals Lorentz violation in an ultracold gas, thus leading to an effective disintegration constant with temperature dependence. To see the temperature effect on half-life time, Eq. (19) is written as

$$
N=N_{0} \exp \left(-\lambda_{T} t\right)=N_{0} \exp \left(-\frac{\lambda t}{\sqrt{1-\frac{T_{\min }}{T}}}\right),
$$

where there emerges an effective disintegration constant $\lambda_{T}$ due to the dilation of the proper time [Eq. (18)] close to the minimum temperature $T_{\min }$ connected to the minimum speed $V$, so that we find $\lambda_{T}=\lambda / \sqrt{1-T_{\min } / T}$, which means an increase of the disintegration constant at lower temperatures, thus leading to the half-life time suppressed by very low temperatures, as

$$
t_{1 / 2}(T)=\frac{\ln (2)}{\lambda_{T}}=\frac{\ln (2)}{\lambda} \sqrt{1-\frac{T_{\min }}{T}},
$$

or simply

$$
t_{1 / 2}^{\prime}=t_{1 / 2} \sqrt{1-\frac{T_{\min }}{T}},
$$

where $t_{1 / 2}(T)=t_{1 / 2}^{\prime}$ is the suppressed half-life time of the ultracold radioactive sample (e.g. $\mathrm{Na}^{25}$ ) and $t_{1 / 2}$ is the well-known half-life time.

The suppressed half-life given in Eq. (22) and shown in Figs. 3 and $\underline{4}$ for two intervals of very low temperatures closer to $10^{-12} \mathrm{~K}$ represents the experimental signature of a Lorentz violation in the infrared regime due to the universal minimum speed $V$.

The equation for the decay law with a half-life time suppression is

$$
\frac{d N}{d t}=-R(T)=-\frac{\lambda}{\sqrt{1-\frac{T_{\min }}{T}}} N,
$$

whose solution is given by Eq. (20), thus leading to a suppression of the half-life time at lower temperatures [Eq. (22)] as it can be seen in Figs. $\underline{3}$ and $\underline{4}$ for $\beta^{-}$ decay of the isotope $\mathrm{Na}^{25} \cdot R(T)=-d N / d t$ is the increased decay rate for lower temperatures. So we write

$$
R(T)=\frac{\lambda}{\sqrt{1-\frac{T_{\min }}{T}}} N .
$$




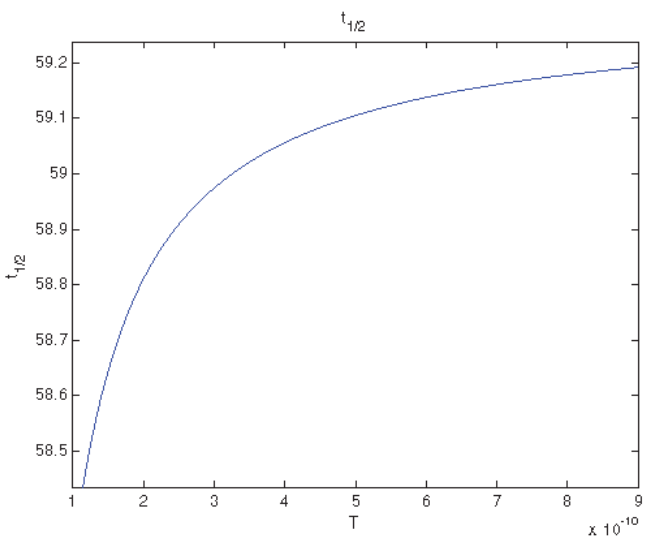

Fig. 3. The graph shows the suppression of the half-life time [Eq. (22)] for $\mathrm{Na}^{25}$ thermalized with an ultracold gas of $\mathrm{Na}^{23} \mathrm{~K}^{40}$ for the range of temperatures $10^{-10} \mathrm{~K} \leq T \leq 10^{-9} \mathrm{~K}$ close to the order of temperature that will be obtained in CAL. It is known that $t_{1 / 2} \cong 59.3 \mathrm{~s}$ for the radioactive sample of $\mathrm{Na}^{25}$ with $\beta^{-}$decay, where $\mathrm{Na}^{25} \rightarrow \beta^{-}+\mathrm{Mg}^{25}$.

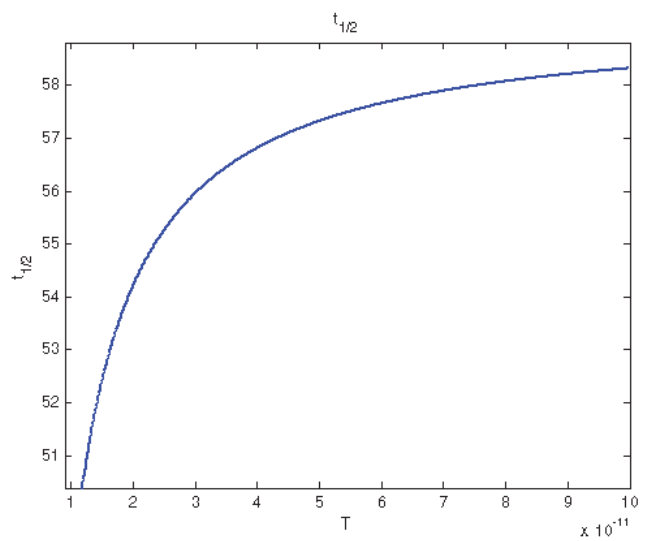

Fig. 4. The graph shows the suppression of the half-life time [Eq. (22)] for $\mathrm{Na}^{25}$ thermalized with an ultracold gas of $\mathrm{Na}^{23} \mathrm{~K}^{40}$ for the range of temperatures with one order below that obtained by the current technology, i.e. $10^{-11} \mathrm{~K} \leq T \leq 10^{-10} \mathrm{~K}$, which will be probably obtained in CAL after a technological improvement.

In Eq. (22), when $T \rightarrow T_{\min }\left(\sim 10^{-12} \mathrm{~K}\right)$, the half-life time of the radioactive sample would become completely suppressed $\left(t_{1 / 2}^{\prime} \rightarrow 0\right)$, but the best technology for cooling will be made soon by NASA in CAL for reaching the order of $10^{-9} \mathrm{~K}$ of a sodium-potassium gas confined during a few seconds. This first experiment could lead to a very slight suppression of the half-life time of a certain radioactive sample thermalized with the sodium-potassium gas $\left(\mathrm{Na}^{23} \mathrm{~K}^{40}\right)$ and thus the detection of such a suppression seems to be difficult at first sight depending on the time resolution of the cooling devices with lasers. However, in the future, the improvement of 
the experiment in CAL for reducing even more the temperature until it reaches the order of $10^{-10} \mathrm{~K}$ with confination of a few hundred seconds will allow to detect a larger suppression of the half-life time as, for instance, a sample of the isotope $\mathrm{Na}^{25}$ whose half-life time is about $t_{1 / 2} \cong 59.3 \mathrm{~s}$ with the usual disintegration constant $\lambda \cong 0.0117 \mathrm{~s}^{-1}$, thus needing a time of confination of a few hundred seconds to be at least of the same order of its half-life time.

\section{Prospects}

The study of the ultracold atoms close to the minimum speed $V$, which has origin in gravity $15(V \propto \sqrt{G})$ could reshape our understanding of matter, dark energy and the fundamental nature of gravity within the scenario of a quantum gravity connected to the cosmological constant. So the experiments that will be made in CAL could give us insight into a new aspect of quantum gravity and dark energy by means of a change in the passing of time predicted by SSR at much lower energies break down Lorentz symmetry, thus leading to the half-life time suppression of an ultracold radioactive sample (e.g. $\mathrm{Na}^{25}$ ) thermalized with an ultracold gas (e.g. $\mathrm{Na}^{23} \mathrm{~K}^{40}$ ). Other radioactive samples or even ultracold gases could be used depending on the most favorable experimental conditions.

\section{References}

1. R. Bluhm, Lect. Notes Phys. 702, 191 (2006), arXiv:hep-ph/0506054.

2. S. M. Carroll, G. B. Field and R. Jackiw, Phys. Rev. D 41, 1231 (1990).

3. V. A. Kostelecky and N. Russell, Rev. Mod. Phys. 83, 11 (2011), arXiv:0801.0287.

4. V. A. Kostelecky and M. Mewes, Phys. Rev. D 66, 056005 (2002).

5. T. Jacobson, S. Liberati and D. Mattingly, Phys. Rev. D 67, 124011 (2003).

6. J. J. Wei and X. F. Wu, Astrophys. J. 851, 127 (2017).

7. C. A. G. Almeida, M. A. Anacleto, F. A. Brito, E. Passos and J. R. L. Santos, arXiv:1712.00995.

8. M. Schreck, arXiv:1711.11167.

9. T. P. Netto, arXiv:1711.05193.

10. T. Prudencio, L. S. Amorim, H. Belich and H. L. C. Louzada, arXiv:1706.04456.

11. L. Nogués et al., arXiv:1710.08342.

12. G. Lambiase and F. Scardigli, arXiv:1709.00637.

13. J. Magueijo and L. Smolin, Phys. Rev. Lett. 88, 190403 (2002).

14. G. A. Camelia, Nature 418, 34 (2002).

15. C. Nassif, Int. J. Mod. Phys. D 25, 1650096 (2016).

16. C. Nassif, Gen. Relat. Gravit. 47, 1 (2015).

17. C. Nassif, Int. J. Mod. Phys. D 21, 1 (2012).

18. C. Nassif, Int. J. Mod. Phys. D 19, 539 (2010).

19. C. Nassif, R. F. Santos and A. C. Amaro de Faria, Jr., Int. J. Mod. Phys. D 27, 1850011 (2018).

20. C. J. Adler, Am. J. Phys. 55, 739 (1987).

21. R. P. Feynman, R. B. Leighton and M. Sands, The Feynman Lectures on Physics, Vol. 1 (Addison-Wesley Reading, 1963), Secs. 15-8 and 16-4.

22. L. V. Okun, Phys. Today 42, 31 (1989).

23. T. R. Sandin, Am. J. Phys. 59, 1032 (1991). 
24. W. Rindler, Introduction to Special Relativity (Clarendon Press, 1982), pp. 79-80; ibid. Essential Relativity, 2nd edn. (Springer, 1977).

25. E. F. Taylor and J. A. Wheeler, Spacetime Physics, 2nd edn. (W. H. Freeman Co., 1992), pp. 246-251.

26. T. Padmanabhan, arXiv:gr-qc/0802.1798.

27. M. Pigneur et al., Phys. Rev. Lett. 120, 173601 (2018). 